\title{
Anomalies in the Low Frequency Vibrational Density of States for a Polymer with Intrinsic Microporosity - The Boson Peak of PIM-1
}

DOI:

10.1039/C7CP07141H

\section{Document Version}

Accepted author manuscript

Link to publication record in Manchester Research Explorer

Citation for published version (APA):

Zorn, R., Yin, H., Lohstroh, W., Harrison, W., Budd, P., Pauw, B. R., Böhning, M., \& Schönhals, A. (2017). Anomalies in the Low Frequency Vibrational Density of States for a Polymer with Intrinsic Microporosity - The Boson Peak of PIM-1. Physical Chemistry Chemical Physics: high quality research in physical chemistry, chemical physics and biophysical chemistry. https://doi.org/10.1039/C7CP07141H

\section{Published in:}

Physical Chemistry Chemical Physics: high quality research in physical chemistry, chemical physics and biophysical chemistry

\section{Citing this paper}

Please note that where the full-text provided on Manchester Research Explorer is the Author Accepted Manuscript or Proof version this may differ from the final Published version. If citing, it is advised that you check and use the publisher's definitive version.

\section{General rights}

Copyright and moral rights for the publications made accessible in the Research Explorer are retained by the authors and/or other copyright owners and it is a condition of accessing publications that users recognise and abide by the legal requirements associated with these rights.

\section{Takedown policy}

If you believe that this document breaches copyright please refer to the University of Manchester's Takedown Procedures [http://man.ac.uk/04Y6Bo] or contact uml.scholarlycommunications@manchester.ac.uk providing relevant details, so we can investigate your claim.

\section{OPEN ACCESS}


Anomalies in the Low Frequency Vibrational Density of States for a Polymer with Intrinsic Microporosity - The Boson Peak of PIM-1

Reiner Zorn ${ }^{1}$, Huajie Yin ${ }^{2}$, Wiebke Lohstroh ${ }^{3}$, Wayne Harrison ${ }^{4}$, Peter M. Budd ${ }^{4}$, Brian R. Pauw $^{2}$, Martin Böhning ${ }^{2}$, Andreas Schönhals ${ }^{2, *}$

${ }^{1}$ Jülich Centre for Neutron Science JCNS and Institute for Complex Systems ICS, Forschungszentrum Jülich, 52425 Jülich, Germany

${ }^{2}$ Bundesanstalt für Materialforschung und -prüfung (BAM), Unter den Eichen 87, 12205

Berlin, Germany.

${ }^{3}$ Heinz Maier-Leibnitz Zentrum (MLZ),Technische Universität München, Lichtenbergstraße 1, 85748 Garching, Germany

${ }^{4}$ The University of Manchester, School of Chemistry, Manchester M13 9PL, United Kingdom 


\begin{abstract}
:
Polymers with intrinsic microporosity are promising candidates for the active separation layer in gas separation membranes. Here, the vibrational density of states (VDOS) for PIM-1, the prototypical polymer with intrinsic microporosity, is investigated by means of inelastic neutron scattering. The results are compared to data measured for a more conventional highperformance polyimide used in gas separation membranes (Matrimid). The measured data show the characteristic low frequency excess contribution to VDOS above the Debye sound wave level, generally known as the Boson peak in glass-forming materials. In comparison to the Boson peak of Matrimid, that of PIM-1 is shifted to lower frequencies. This shift is discussed considering the microporous, sponge-like structure of PIM-1 as providing a higher compressibility at the molecular scale than for conventional polymers. For an annealed PIM-1 sample, the Boson Peak shifts to lower frequencies in comparison to the un-annealed sample. These changes in the VDOS of the annealed PIM-1 sample are related to changes in the microporous structure as confirmed by X-ray scattering.
\end{abstract}




\section{Introduction}

A key technology for the efficient separation of gases in the energy supply and chemical industries is membrane technology, which stands out as a greener technology compared to more energy consuming techniques based on cryogenic or adsorption processes. Glassy polymers are still the most important class of materials for the active separation layer in such applications, due to the easy and inexpensive processability of polymers at large scales. Nearly 15 years ago, a new class of suitable polymeric materials appeared which are completely soluble in conventional solvents but characterized by an intrinsic microporosity (PIM), firstly synthesized as PIM-1 (a polybenzodioxane) by Budd and McKeown. ${ }^{1,2}$ The intrinsic microporosity manifests itself by a rather high BET surface $>700 \mathrm{~m}^{2} / \mathrm{g}$ determined by low temperature $\mathrm{N}_{2}$ adsorption. ${ }^{2}$ Conventional glassy high-performance polymers used for gas separation membranes typically possess a large fractional free volume, which leads to extremely high gas permeabilities but at the same time often to insufficient permselectivity values. One example of the latter case is the polyacetylene PTMSP (see for instance reference [ 3 ]). In contrast, PIMs combine comparably high permeabilities with reasonable permselectivities. For that reasons PIMs are currently considered as state-of-the-art materials for air separation and also hydrogen recovery., ${ }^{4,5}$

No glass transition temperature $\mathrm{T}_{\mathrm{g}}$ could be detected for PIMs with conventional DSC measurements before degradation. At room temperatures PIMs are thus deeply in a glassy state and therefore undergo distinct physical aging effects ${ }^{6}$ which hamper their practical application in gas separation membranes. PIMs share this unwanted property with other glassy or superglassy polymers like polyimides (see for instance $[7,8,9]$ ) or polynorbornenes with stiff mains chains and bulky side groups ${ }^{10}$. Therefore, one current research topic in this field is to reduce physical aging effects either by improved chemical structures or by adding nanofillers (see for instance [11-14]). 
Recently, the molecular mobility and the conductivity of PIM-1 were investigated by broadband dielectric spectroscopy. ${ }^{15}$ In principle a correlation between the molecular mobility and the diffusion of small molecules like gases can be expected for dense polymer systems from transport models ${ }^{16,17}$ as well as from molecular dynamic simulations ${ }^{18,19}$ for conventional glassy polymers. Such a correlation was inferred from experimental data including dielectric results. ${ }^{20,21,22}$ Because the transport mechanism is somewhat different for superglassy polymers with intrinsic microporosity, such a correlation is no longer expected for PIMs. This is supported by atomistic molecular dynamic simulations, where for these systems a continuous free volume phase is proposed. ${ }^{23,24}$ The structure might be considered as consisting of lager pore-like objects, with a broader distribution interconnected by smaller or wider channels. As discussed above, BET surface areas of $720-850 \mathrm{~m}^{2} / \mathrm{g}$ and pore sizes in the range of 5.2 to $10.7 \AA$ are obtained experimentally. ${ }^{6,25,26}$ It is worth to mention that these values are obtained by the application of classical models, which are not especially optimized for PIMs, to BET or positron annihilation data. Moreover, these values depend strongly on processing conditions, like casting solvent and thermal history, as well as methanol treatment or gas exposure. ${ }^{11,26}$ Furthermore, one has to note that these values are also dependent on the characterization technique employed and on the way the data are evaluated. ${ }^{6,27}$

Glassy polymers - as amorphous materials in general - show excess contributions in the vibrational density of states (VDOS; $g(\omega), \omega$ - frequency) in the frequency range of $\omega=0.2-1$ $\mathrm{THz}$ (energy range 1-5 meV) in comparison to what is expected from the Debye model of sound waves, which gives $g(\omega) \sim \omega^{2}$. Therefore, in the reduced representation $g(\omega) / \omega^{2}$ versus frequency $\omega$ a peak is observed, which is known as the Boson Peak (BP) ${ }^{28}$ Generally, the BP is considered as a universal feature of amorphous materials and materials with partial disorder. There are strong indications that the Boson Peak has some relevance for the glass transition despite the fact that the underlying time scales are different (i.e. about $100 \mathrm{~s}$ for the thermal glass transition at $\left.\mathrm{T}_{\mathrm{g}} ; \mathrm{BP}-\mathrm{ps}\right) .{ }^{28}$ Here it is also worth to note that the excitations 
responsible for the Boson Peak are also reflected in an excess contribution to the temperature dependence of the specific heat $c_{p}$ at low temperatures in comparison to the prediction $c_{p} \sim T^{3}$ obtained in Einstein/Debye theory of the specific heat for solids.

Until now, the molecular origin of the Boson Peak has been a subject of controversy. Existing theories can be roughly divided into two classes. In the first class, the BP is considered to originate from quasi-localized modes ${ }^{29-31}$ due to peculiarities of the interatomic forces in the amorphous structure (groups of atoms subjected to a soft potential) and therefore considered to be different from sound waves. The second class of theoretical approaches considers the BP in amorphous materials to be a frequency broadened version of the Van Hove-singularity of crystalline systems. ${ }^{32}$ It is concluded that the VDOS of the amorphous system is in principle related to sound waves and is a modification of the vibrational density of states of the corresponding crystalline system due to random fluctuations of force constants. ${ }^{33}$ Recently, it was demonstrated by numerical simulation that the BP can also be considered to be a consequence of a local breaking of center-inversion symmetry. ${ }^{34}$ Results obtained from studies investigating the VDOS for low molecular weight materials and polymers in nanoscale confinement ${ }^{35-38}$ provide some evidence that the BP is related to collective effects like sound waves. ${ }^{39}$

Here, the vibrational density of states of PIM-1 is explored by inelastic neutron scattering for the first time. Firstly, it has to be clarified if PIM-1 shows a Boson Peak at all, as no glass transition could be detected by conventional thermal analysis up to now. Secondly, in the case that a BP is found and assuming that it is related to sound waves, the propagation of sound waves should be different for a material with a continuous free volume phase (evidenced by molecular simulations ${ }^{23,24}$ ) compared to a polymer with no intrinsic microporosity like a polyimide. If differences in the VDOS are proven to exist for PIM-1 in contrast to a conventional polymer, this would provide further evidence that the BP is related to sound 
waves. Furthermore, the influence of a thermal treatment (i.e. physical ageing) of PIM-1 on the VDOS is investigated.

\section{Experimental:}

The chemical structure of PIM-1 is shown in Figure 1a. The synthesis of PIM-1 was discussed in more detail in ref. 15. Size exclusion chromatography using chloroform as solvent and polystyrenes as calibration standards results in a molecular weight $\mathrm{M}_{\mathrm{W}}$ of $82800 \mathrm{~g} / \mathrm{mol}$ and a polydispersity index of 2.8. The conditions for film formation were discussed in detail also in ref. 15. In brief, $1 \mathrm{~g}$ of PIM-1 was dissolved in chloroform by shaking for 4 to 5 hours. The solution was filtered (5 $\mu \mathrm{m}$ PTFE-filter) and ultra-sonicated for $5 \mathrm{~min}$. Finally, the solution was cast in a Teflon mold, after which it was placed in a closed chamber. To control the solvent evaporation from the film the atmosphere of the chamber was saturated with chloroform. At room temperature, a solid film is formed overnight. It is worth to note, that different annealing and/or conditioning protocols have been discussed in the literature. Different annealing temperatures in the range from $40{ }^{\circ} \mathrm{C}$ to $100{ }^{\circ} \mathrm{C}$ have been employed, which result in different PIM-1 structures having quite different gas permeability values. ${ }^{11,40,41}$ In order to enable comparison of the data, the same annealing protocol was applied as in an earlier study addressing dielectric spectroscopy. ${ }^{15}$ After removing the film from the Teflon mold, it was annealed in a vacuum oven at $75^{\circ} \mathrm{C}$ for 3 days.

To compare the data obtained for PIM-1 with data of a polymer with no intrinsic microporosity a second material was included in this study. For that purpose, Matrimid was chosen as a commercially available high-performance polyimide with no intrinsic microporosity (i.e. no significant BET surface) but with a high free volume, which is frequently used in gas separation applications as well as in studies addressing gas transport and related properties. ${ }^{7,8}$ The chemical structure is given in Figure $1 \mathrm{~b}$. Matrimid $5218\left(3,3^{\prime}-\right.$ 4,4'-benzophenone tetra-carboxylic dianhydride and diaminophenylindane) was obtained 
from Huntsman International LLC. According to the producer it was provided in the fully imidized state. The film preparation followed the same route as described above for PIM-1.

The samples were prepared just before the measurement, for which they were sealed hermetically (by welding) in a flat aluminum cell which is transparent to neutrons.

\begin{tabular}{|l|l|}
\hline Figure 1: Chemical structure of PIM-1 (a) and Matrimid (b). \\
\hline
\end{tabular}

The neutron scattering measurements were performed at the multi-chopper cold neutron timeof-flight spectrometer TOFTOF ${ }^{42}$, operated by the Technische Universität München at the Heinz Maier-Leibnitz Zentrum (MLZ) in Garching, Germany. TOFTOF was used in standard configuration with an incident wavelength of $\lambda_{n}=5.0 \AA$ corresponding to a resolution of $85-$ $100 \mu \mathrm{eV}$ (full width at half maximum) in the q-range used for the VDOS evaluation. In this configuration, the maximal accessible elastic scattering vector is $\mathrm{q}=2.3 \AA^{-1}$. For background and absorption correction of the raw data, the program INX was used. ${ }^{43}$ To obtain the resolution of the instrument the sample was measured at $4 \mathrm{~K}$ because it is expected that all molecular motions are frozen at this low temperature except for the quantum-mechanical zero-point motion. The VDOS was measured at $100 \mathrm{~K}$ to avoid the inelastic scattering due to the vibrations being overlapped by quasielastic contributions. To correct the data for the scattering of the empty can, the empty sample holder was also measured at 4 and $100 \mathrm{~K}$. The program INX was again used to obtain absolute values of the dynamic structure factor. ${ }^{43}$

The SAXS/WAXS measurements were performed on a Xenocs Nano-inXider-SW, with a microfocus X-ray tube with a copper target. The X-rays are focused and monochromatized to a wavelength of $0.154 \mathrm{~nm}$, by means of a multilayer optic, after which they are collimated 
using two scatterless pinholes. The WAXS detector consists of one single-photon counting Pilatus 100k detector module, $93 \mathrm{~mm}$ from the sample, collecting an angular range of $3.1 \mathrm{~nm}^{-1}$ $<\mathrm{q}<4.2 \mathrm{~nm}^{-1}$. The SAXS detector consists of an identical module mounted $933 \mathrm{~mm}$ from the sample behind a semitransparent beamstop, aligned so that a practical angular range of $0.06<$ $\mathrm{q}(1 / \mathrm{nm})<3.6$ could be covered. Data from both detectors have been processed using the DAWN software package ${ }^{44}$, according to the latest available procedures. ${ }^{45}$

The data have been (in the following order) masked, corrected for counting time, darkcurrent, transmission, primary beam flux, background, thickness and solid angle, scaled to absolute units, followed by an azimuthal integration step.

Uncertainties have been calculated by propagating the Poisson statistics through the corrections. Distance- orientation- and beam-center calibration of the SAXS and WAXS detectors were performed using silver behenate and $\mathrm{LaB}_{6}$, respectively. Intensity calibration was achieved using the NIST SRM3600 calibration standard. Without arbitrary scaling, the data from the two detectors overlap with good agreement. It should be noted the SAXS and WAXS parts have different resolutions with respect to $\mathrm{q}$.

\section{Results and Discussion:}

The structure of solid PIM-1 was discussed earlier in detail in refs. [46 and 47] employing a combination of small- and wide-angle X-ray scattering (SAXS/WAXS). X-ray measurements were carried out here for the following two reasons. Firstly, here the sample was cast and annealed in a different way than described in references [46,47]. Secondly, two kinds of inelastic neutron experiments are carried out. The first experiment was carried out on a freshly prepared sample. The second experiment was conducted on the same sample which was subjected to physical aging at higher temperatures. The observed changes in the vibrational density of states were related structural to features revealed by X-ray measurements. Nevertheless all interpretations and assignments made for X-ray result for PIM-1 given here 
are based on discussions in references [46,47] which are supported by models and atomistic molecular simulations.

Figure 2 depicts the SAXS/WAXS pattern of the PIM-1 used in this study in comparison to Matrimid.

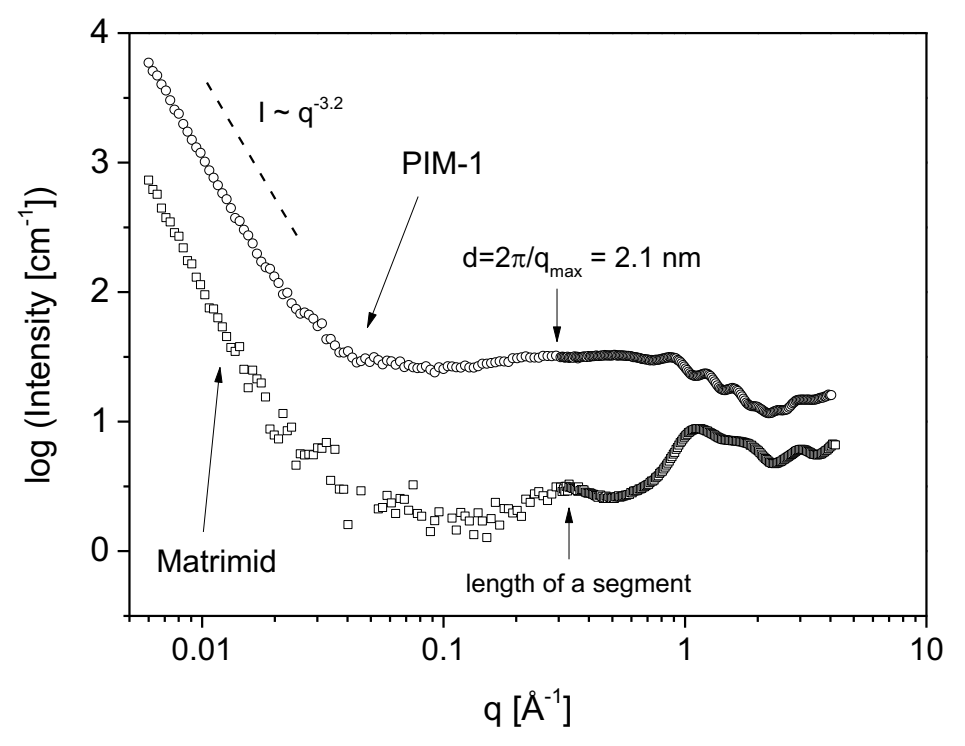

Figure 2: SAXS/WAXS pattern for PIM-1 (circles) and Matrimid (squares). The curve for Matrimid is by half a decade shifted along the y-axis to lower values for clarity.

The observed X-ray pattern of PIM-1 shows similar features to that reported in refs. [46] and [47]. The observed small differences may be due to a different preparation and annealing protocol used in this study. For PIM-1 at low q-values the intensity decreases following a power law with an exponent of $3.2 \pm 0.2$. This seems to be in disagreement with a dense mass fractal (exponent 3) but with a structure with a fractal surface (exponent 3.2), ${ }^{48}$ discussed also in ref. [47]. This interpretation is also in agreement with a possible continuous free-volume phase of PIM-1. For Matrimid a behavior close to the classical Porod law (with an intensity decay of approximately $\mathrm{I} \sim \mathrm{q}^{-4}$ ) is obtained as found for most polymers.

In the q-range from $0.05 \AA^{-1}$ to $1 \AA^{-1}$ for PIM-1 a broad scattering feature is observed with weak maximum at ca. $\mathrm{q}_{\max }=0.29 \AA^{-1}$. Applying a simple Bragg model as rough approximation, this would correspond to a mean distance of ca. $2.1 \mathrm{~nm}$. As discussed in ref. 
[47] the maximum pore size observed by various techniques is $1 \mathrm{~nm}$. Nevertheless, it is pointed out that the scattering in the range between $0.05 \AA^{-1}$ and $1 \AA^{-1}$ is most likely related to the microposity ${ }^{46}$ because it is sensitive to the annealing (or physical ageing) of PIM-1, which in turn leads to a reduction of the permeability probably due to a reduced diffusion coefficient resulting from a partial collapse of the microporous structure. ${ }^{47}$ The scattering in the $\mathrm{q}$ range between $0.05 \AA^{-1}$ and $0.1 \AA^{-1}$ evidence larger scattering objects which might be related to a continuous free volume phase suggested by atomistic molecular dynamic simulations. ${ }^{23,24}$ Matrimid shows a slight peak at $\mathrm{q}=0.3 \AA^{-1}$. This cannot be related to any kind of microporosity because Matrimid is a polymer without intrinsic microporosity and was especially selected for comparison for that reason. The origins for this peak are not clear up to now. Nevertheless, it is interesting to note that the corresponding Bragg distance of the peak of ca. $1.9 \mathrm{~nm}$ corresponds roughly to the length of the monomer unit of Matrimid, which is estimated to be about $2 \mathrm{~nm}$ from simple geometric considerations.

In the higher q-range $\left(q \geq 1 \AA^{-1}\right)$, a scattering behavior is observed for Matrimid, which is expected for amorphous polymers, consisting of an amorphous halo due to correlations of carbon atoms and some broad structural features. This is considerably different for PIM-1, where a broad structural feature is observed with at least four weak scattering peaks. Up to now it has not been completely clear which structures are responsible for these scattering peaks. On the one hand, they might be related to an underlying form factor in addition to the structure factor related to the microporosity. On the other hand, atomistic molecular dynamics (MD) simulations show that these high-q features might originate from correlations due to characteristic distances between spiro centers of different chains. ${ }^{46}$

Neutron spectroscopy senses the molecular motions on microscopic length and time scales. ${ }^{49}$ During the scattering experiment momentum and energy are exchanged between neutrons and the nuclei of the sample, yielding information about the space and time dependence of these 
dynamical processes. As the main experimental quantity, the double differential cross section is obtained, which is given as

$$
\frac{d^{2} \sigma}{d \Omega d(\hbar \omega)}=\frac{1}{4 \pi} \frac{k_{f}}{k_{i}}\left(\sigma_{c o h} S_{c o h}(q, \omega)+\sigma_{i n c} S_{i n c}(q, \omega)\right)
$$

Here $\mathrm{k}_{\mathrm{i}}$ and $\mathrm{k}_{\mathrm{f}}$ are the incident and final wave vectors of the neutron beam and $\mathrm{q}$ denotes the scattering vector. Here $\hbar \omega$ is the energy transfer $\Delta \mathrm{E}$ related to an angular frequency $\omega$ where $\hbar$ is the Planck constant divided by $2 \pi(\hbar=h / 2 \pi)$, and $\Omega$ is the solid angle of detection. $S_{\text {inc }}(\mathrm{q}, \omega)$ is the incoherent dynamic structure factor related to the self-correlation function of nuclei where $S_{c o h}(q, \omega)$ is the coherent dynamic structure factor related to pair correlations of nuclei. $\sigma_{\mathrm{coh}}=4 \pi \overline{\mathrm{b}}^{2}$ and $\sigma_{\mathrm{inc}}=4 \pi\left(\overline{\mathrm{b}^{2}}-\overline{\mathrm{b}}^{2}\right)$ are the scattering cross-sections for coherent and incoherent scattering where $\mathrm{b}$ are the scattering lengths averaged over isotopes and spin disorder. (The coherent part in this equation is strictly correct only for elemental samples. But since the actual sample scatters predominantly incoherently this simplification is not relevant for the calculation of the VDOS.) The calculated scattering cross-sections for PIM-1 are $\sigma_{\text {inc,PIM-1 }}=$ $0.278 \mathrm{~mm}^{-1}$ and $\sigma_{\text {coh,PIM-1 }}=0.0376 \mathrm{~mm}^{-1}$. The corresponding values for Matrimid are $\sigma_{\text {inc,Mat }}=$ $0.281 \mathrm{~mm}^{-1}$ and $\sigma_{\mathrm{coh}, \mathrm{Mat}}=0.0381 \mathrm{~mm}^{-1}$. The calculation is given in the Supporting Information. Therefore, the observed scattering is primarily incoherent due to the hydrogen nuclei.

Figure 3a shows spectra for Matrimid as measured by TOFTOF normalized to the height of the elastic peak at two different temperatures at an angle of $122^{\circ}$. The spectrum measured at $4 \mathrm{~K}$ serves as resolution. The data obtained at $100 \mathrm{~K}$ show a Boson Peak around an energy transfer of ca. $1.3 \mathrm{meV}$. Figure $3 \mathrm{~b}$ gives corresponding data for PIM-1. Compared to Matrimid, here the Boson Peak appears at lower energy transfer values below $1 \mathrm{meV}$.

In order to extract the low-energy vibrations from the raw data, the incoherent dynamic scattering function is expressed by the standard expression for the one-phonon scattering: ${ }^{50}$ 
$\mathrm{S}(\mathrm{q}, \omega)=\mathrm{e}^{-2 \mathrm{~W}(\mathrm{q})}\left(\delta(\omega)+\frac{\hbar \mathrm{q}^{2}}{2 \overline{\mathrm{m}}} \frac{\mathrm{g}(\omega)}{-\omega} \times\left(1-\exp \left(\frac{\hbar \omega}{\mathrm{k}_{\mathrm{B}} \mathrm{T}}\right)\right)^{-1}\right)$

Here $\mathrm{e}^{-2 \mathrm{~W}(\mathrm{q})}$ is the Debye-Waller factor and $\mathrm{k}_{\mathrm{B}}$ is the Boltzmann constant. $\overline{\mathrm{m}}$ denotes the average mass of an atom. Due to the high incoherent neutron scattering cross section the influence of hydrogen atoms is overrepresented in this expression. For the calculation of $\overline{\mathrm{m}}$ it is assumed that in the frequency region studied hydrogen atoms participate proportionately in the total vibrational spectrum of the materials. Generally, the measured scattering is the convolution of Equ. (2) with the resolution function of the instrument $\mathrm{R}(\mathrm{q}, \omega)$. The Boson Peak is a broad feature in comparison to the resolution. Therefore, the convolution effect on the inelastic term can be omitted and it holds: ${ }^{51}$

$\mathrm{S}_{\mathrm{obs}}(\mathrm{q}, \omega)=\mathrm{R}(\mathrm{q}, \omega) \otimes \mathrm{S}(\mathrm{q}, \omega) \approx \mathrm{e}^{-2 \mathrm{~W}(\mathrm{q})}\left(\mathrm{R}(\mathrm{q}, \omega)+\frac{\hbar \mathrm{q}^{2}}{2 \overline{\mathrm{m}}} \frac{\mathrm{g}(\omega)}{-\omega} \times\left(1-\exp \left(\frac{\hbar \omega}{\mathrm{k}_{\mathrm{B}} \mathrm{T}}\right)\right)^{-1}\right)$

By measuring the spectra at two different temperatures (here $4 \mathrm{~K}$ and $100 \mathrm{~K}$ ) a system of two linear equations is obtained. From that system both the vibrational density of states $g(\omega)$ and the resolution of the instrument $\mathrm{R}(\mathrm{q}, \omega)$ can be obtained. The resulting VDOS (which in principle should be q-independent) was averaged over the range $\mathrm{q}=1.5-2.3 \AA^{-1}$ to improve accuracy.

It has to be noted that below about $0.8 \mathrm{ps}^{-1}$ all VDOS curves show a strong increase towards zero frequency. This feature has been found in some but not all VDOS calculations from inelastic neutron scattering. It could be either due to quasielastic scattering from processes with very low energy barriers or yet unknown excitations with energies below that of the BP. 


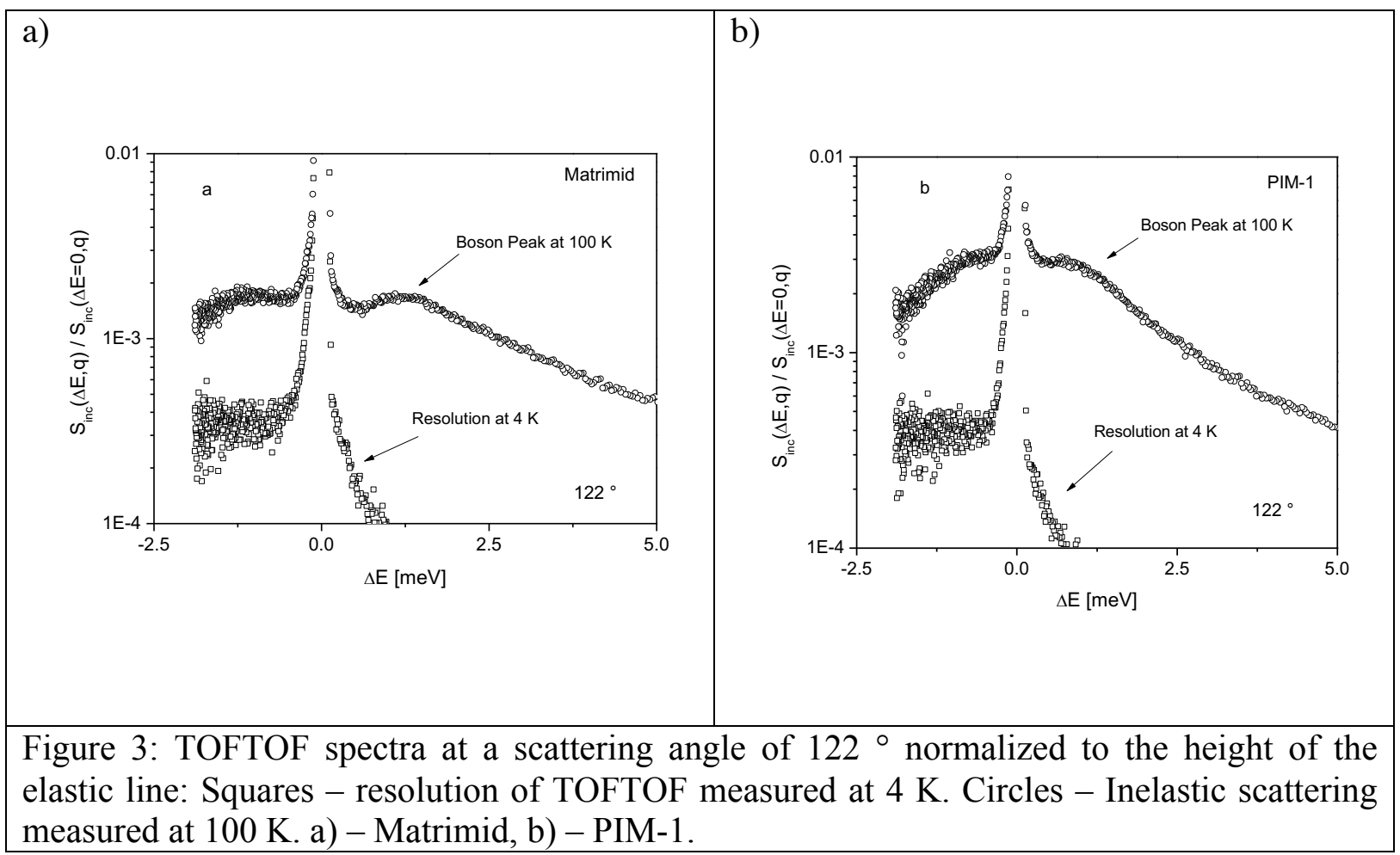

In Figure 4 the density of states $g(\omega)$ normalized by $\omega^{2}$ (Debye density of states) of Matrimid and Poly(methyl phenyl siloxane) (PMPS) are compared, where the data for PMPS were taken from ref. [35]. This figure evidences that Matrimid has a Boson peak as expected from the raw data. Its frequency position is similar to the VDOS of PMPS. Similar results than for PMPS are also found for other conventional amorphous polymers like polystyrene ${ }^{52,53}$ or poly(methyl methacrylate) ${ }^{54}$ Therefore, it can be concluded the BP observed for Matrimid resembles the behavior of conventional polymers in the glassy state. 


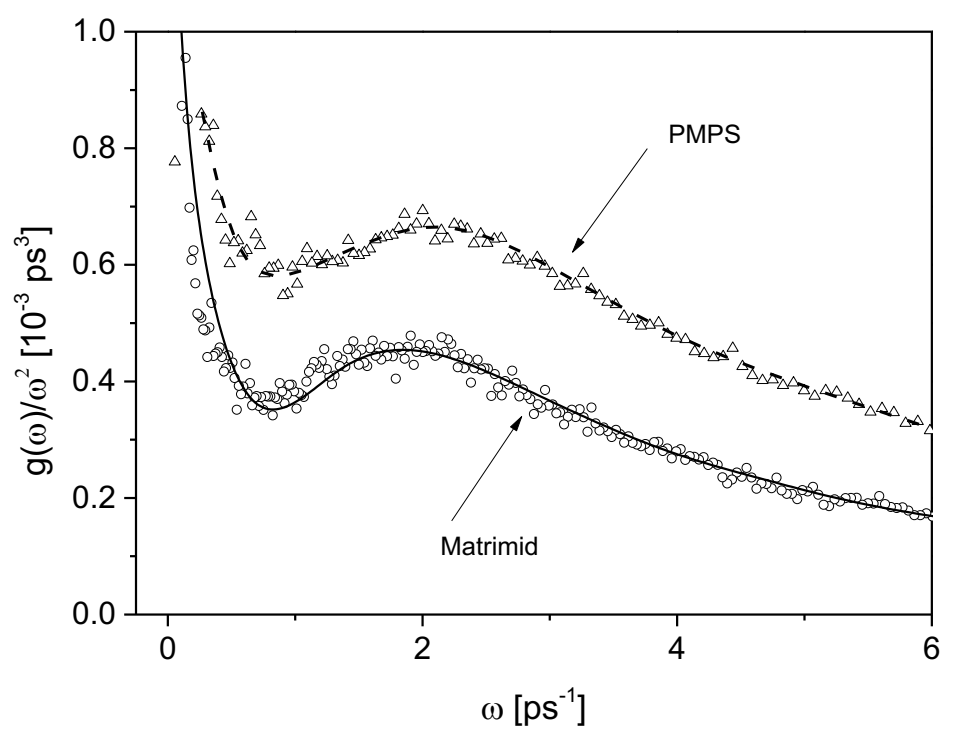

Figure 4: Vibrational density of states normalized by $\omega^{2}$ for Matrimid (circles) and poly(methyl phenyl siloxane) (triangles) obtained from the q range $1.5-2.3 \AA^{-1}$ of the TOFTOF data. The data for PMPS were taken from ref. [35] (from $q=1.55-2.05 \AA^{-1}$ ). Lines are guides for the eyes.

In Figure 5 the VDOS normalized by $\omega^{2}$ is compared for Matrimid and PIM-1. A Boson Peak is also observed for PIM-1. Surprisingly, for PIM-1 the BP is shifted to lower frequencies and gains in intensity compared to that of Matrimid. In the class of theories relating the Boson Peak to sound waves an elastic continuum is considered which is characterized by an elastic modulus which is allowed to fluctuate due to the amorphous structure. ${ }^{55}$ These theories predict a shift of the BP to lower frequencies for a softer material on a molecular scale. This concept was supported by experiments were the stiffness of the material under investigation was varied either by mixing ${ }^{56}$ or changing its structure in a continuous way. ${ }^{57}$ At first glance the shift of the Boson Peak to lower frequencies seems to be in contradiction to the stiff chain structure of PIM-1 with ladder-like structure and a spiro center (see Figure 1a). A more detailed consideration leads to the conclusion that the structure of PIM-1 decreases dramatically the number of conformational degrees of freedoms of a corresponding chain by suppressing the possibilities of segmental rotations (see Figure 1a). This is different for Matrimid, where some degrees of segment rotations remain due to the existence of single 
bonds in the polymer backbone. The stiff backbone structure of PIM-1 leads to an early solidification during film formation by solvent evaporation, and therefore results in a loose structure of the solid film. Such a "sponge-like" structure allows a higher compressibility, at a length scale defined by the lowest q value considered, than the more densely packed structure of the conventional Matrimid with no microporosity. (The relationship of the dynamic structure factor to a generalized $\mathrm{q}$ and frequency dependent compressibility was discussed in ref. [58].) This structure results in a kind of 'softened' matrix of PIM-1 compared to Matrimid and therefore gives rise to a shift of the BP to lower frequencies. This line of argumentation is also consistent with the experimental results that PIM-1 has a lower elastic modulus (ca. 1 GPa, see reference [59]) than Matrimid (ca. 2.7 GPa, see reference [60]).

A similar result was found recently on a set of pressurised glasses. The Boson Peak scales with the Debye energy $E_{D}$. $E_{D}$ in turn is proportional to the effective sound velocity which is dominated by the transverse contribution. ${ }^{61}$

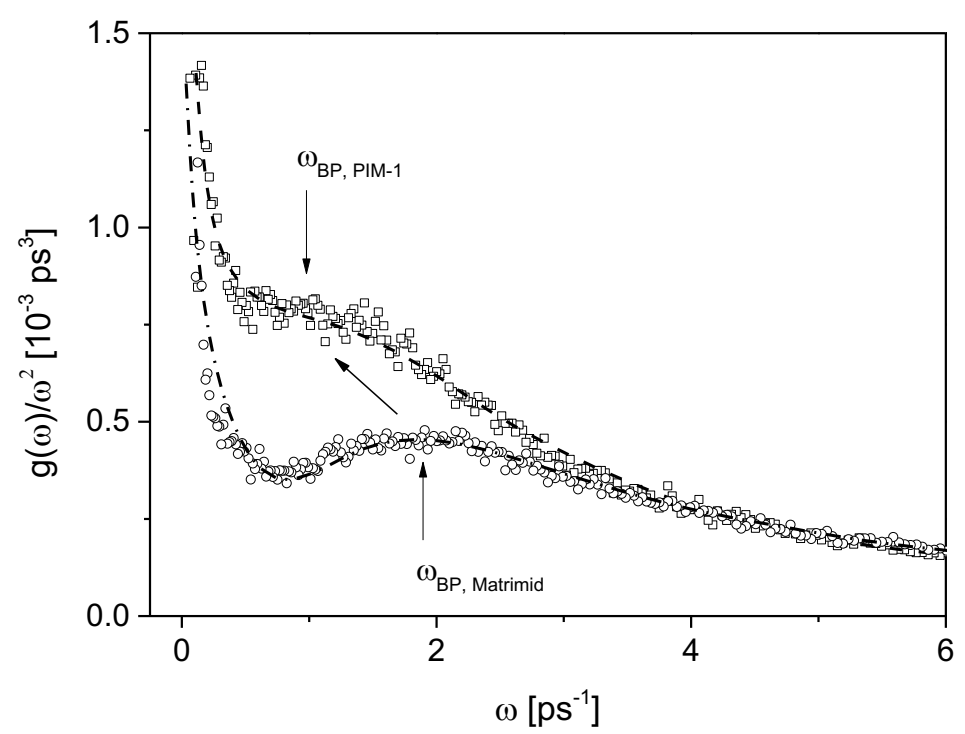

Figure 5: Vibrational density of states normalized by $\omega^{2}$ for Matrimid (circles) and PIM-1 (squares) obtained from the $\mathrm{q}$ range $1.5-2.3 \AA^{-1}$ of the TOFTOF data. Lines are guides for the eyes. The increase in the VDOS for frequencies lower than $0.5 \mathrm{ps}^{-1}$ is related to contributions of quasielastic contributions may by methyl group rotations. 
Sokolov et al. ${ }^{62-64}$ related the maximum frequency of the Boson Peak $\omega_{\mathrm{BP}}$ to a length scale characteristic for glasses $\xi$ by

$\xi=\mathrm{Ac}_{\mathrm{t}} / \omega_{\mathrm{BP}}$

where $A$ is a constant $(A \approx 1)$ and $c_{t}$ is the transversal sound velocity. In principle the transverse sound velocity can be measured by Brillouin scattering (see for instance ref. 65). But for these measurements a fully transparent sample is required which does not show any adsorption or fluorescence. Unfortunately the color of a cast PIM-1 is yellowish which means that PIM-1 absorbs in the blue wave length range of light (see Supporting Information). Moreover PIM-1 is fluorescent. These properties of PIM-1 prevent Brillouin scattering experiments. Although $\mathrm{c}_{\mathrm{t}}$ is not known for PIM-1, but assuming that a similar length scale is relevant to both PIM-1 and Matrimid the lower maximum frequency of the BP for PIM-1 would mean that the sound velocity is lower for PIM-1 in comparison to Matrimid. From a simple intuitive picture it should be expected that the sound velocity should be higher in a more dense material than for a sample with lower density. This is in agreement with the density values found for PIM-1 (1.15 $\mathrm{g} \mathrm{cm}^{-3}$, see ref. [14]) and Matrimid (1.24 $\mathrm{g} \mathrm{cm}^{-3}$, see ref. [9]) as well as with the considerations given above.

To study the influence of physical aging on the Boson Peak of PIM-1 the sample was heated twice with a heating rate of $0.45 \mathrm{~K} / \mathrm{min}$ to $525 \mathrm{~K}$ and cooled down. It should be noted that TGA measurements show that PIM-1 does not decompose chemically or crosslinked at 525 $\mathrm{K}$. This was also confirmed by a dielectric study were the sample was exposed to similar high temperatures comparable time. ${ }^{15}$ After this dielectric experiment the sample was fully soluble and a new film could be cased with identical properties. 


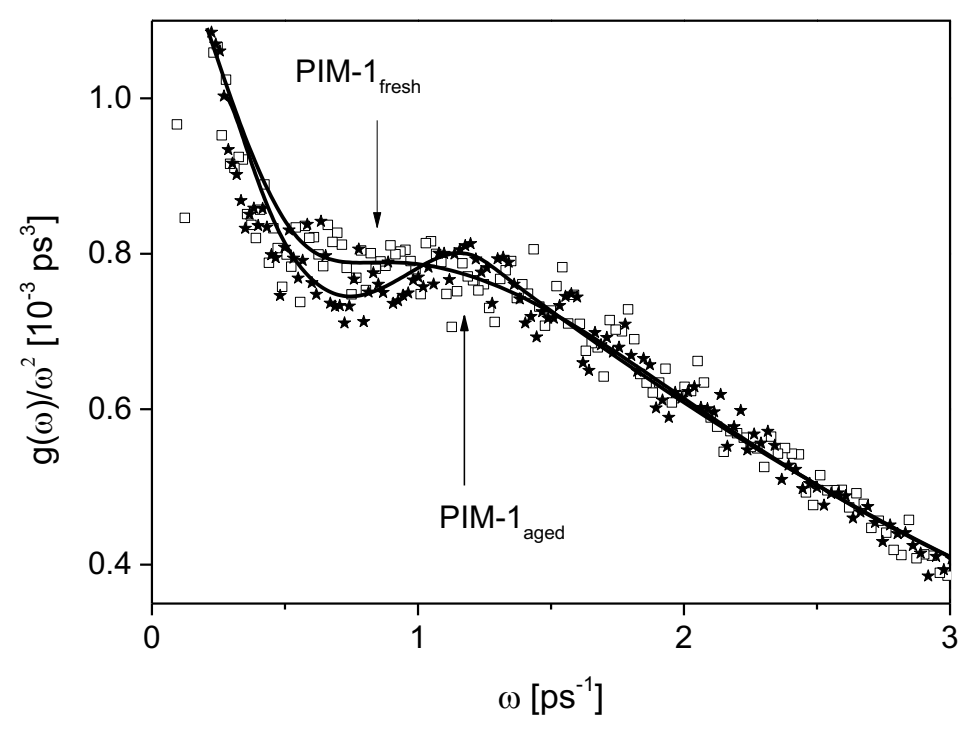

Figure 6: Vibrational density of states normalized by $\omega^{2}$ for PIM-1: freshly prepared squares; aged - asterisk. Lines are guides for the eyes.

In Figure 6 the BP for the freshly prepared sample is compared with the aged one. For the aged sample the BP shifts to higher frequencies. It has to be noted that the observed difference in the data for the freshly prepared sample and the aged one is significant. This becomes clear comparing the VDOS of a fresh sample of Matrimid and a sample which was aged in a similar way as PIM-1 (see inset Fig. 7). In the case of Matrimid the data for the fresh and the aged sample collapse completely into one curve.

In the line of argumentation discussed above and also assuming that the Boson Peak is related to sound waves, a shift of the BP to higher frequencies would mean that PIM-1 becomes 'harder' on length scales defined by the lowest employed q value. Such an interpretation is in complete agreement with a partial collapse of the microporous structure during the physical aging of PIM-1. A partial collapse of the microporous pore structure was experimentally evidenced by a dramatic decrease of the permeability values of PIM-1. In refs. 46 and 47 the partial collapse of the microporous pore structure was discussed as a combined effect of the pore volume fraction (changes of pore shape and pore size distribution) and a shrinking of pores by analyzing X-ray data in a quantitative way. Most importantly, a decrease of the 
fraction of micropores was deduced from the X-ray data. ${ }^{47}$ This decreasing fraction of micropores leads to a densification of PIM-1 during physical aging. In turn this process decreases the compressibility and increases the sound velocity resulting in a shift of the BP to higher frequencies.

Figure 7 compares the Boson Peak of the aged PIM-1 with that of the aged Matrimid sample. This comparison reveals that the Boson Peak for aged PIM-1 is much narrower than that for fresh and aged Matrimid.

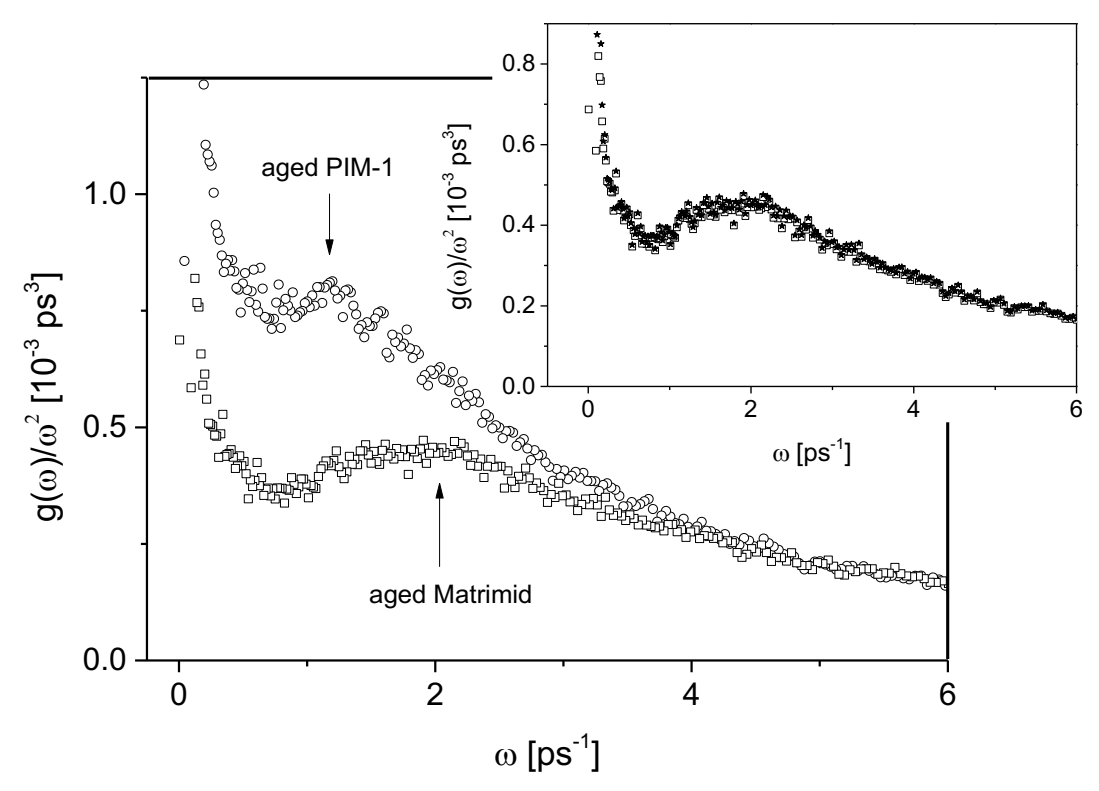

Figure 7: Vibrational density of states normalized by $\omega^{2}$ for aged PIM-1 (circles) and aged Matrimid (squares). The inset compares the VDOS for a freshly prepared (squares) and an aged (stars) Matrimid sample.

This also becomes clear when comparing the BP for the freshly prepared and the aged PIM-1 sample (Figure 6). The Boson Peak for the aged sample is much narrower than that of freshly prepared PIM-1. Within the picture of sound waves this can be understood as that during the aging process the low frequency modes are cut out first. A similar interpretation was employed for changes of the Boson Peak for confined polymers ${ }^{35,36}$. This would mean that larger pores shrink first to smaller ones during the aging process, in agreement with an increase of the intensity of $g(\omega)$ at higher frequencies leading to a sharper BP. 
To confirm the change in the microporous structure for the aged PIM-1 sample in comparison to the freshly prepared one, X-ray measurements were carried out on a sample which was aged in a similar way as employed in the neutron scattering experiments (Figure 8). Changes in the X-ray pattern take place in the $\mathrm{q}$ range between 0.2 and $0.8 \AA^{-1}$. These changes are similar to those reported in ref. [47] but not as pronounced. This is due to the shorter annealing time applied here. The changes were observed in the wave vector region which is sensitive to the microporous structure, as discussed above. Therefore they provide strong evidence that the changes observed in the Boson peak are directly related to changes in the microporous structure of PIM-1.

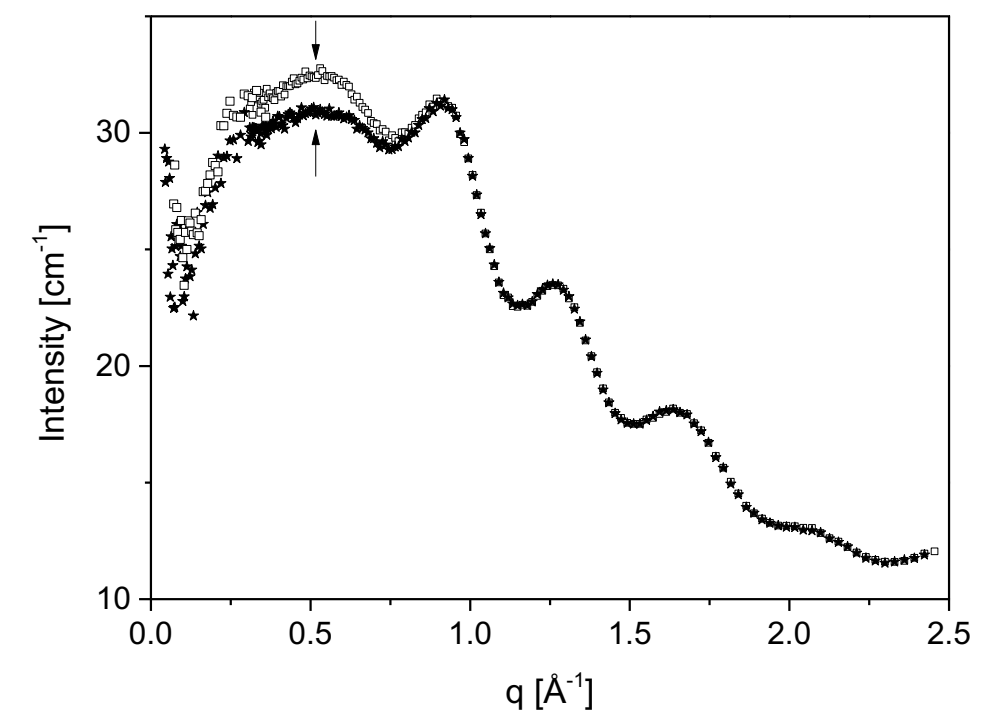

Figure 8: X-ray pattern for PIM-1: freshly prepared - squares; aged - asterisk. Lines are guides for the eyes.

\section{Conclusions}

Inelastic neutron scattering is employed utilizing a Time-of-Flight spectrometer (TOFTOF at the MLZ) to measure the vibrational density of states (VDOS) for PIM-1, which is the archetypal polymer with intrinsic microporosity (PIM). Polymers with intrinsic microporosity are considered possible candidates as materials for the active separation layer in gas separation membranes, massively improving their performance compared to currently used 
polymers. As PIM-1 is a (super-) glassy polymer, the question arises whether or not PIM-1 shows the characteristic excess contributions in the VDOS to what is expected from the Debye theory of sound waves, known as the Boson Peak for other glass-forming materials. In addition to the measurements carried out for PIM-1, experiments on a standard polymer for gas separation membranes (Matrimid) are also carried out for comparison, with the distinct difference that Matrimid has no intrinsic microporosity. The measurements revealed that PIM-1 shows a Boson Peak, which it is shifted to essentially lower frequencies compared to that of Matrimid. Assuming the Boson peak is due to sound waves, this shift to lower frequencies in comparison to a conventional glassy polymer points to a softer structure of PIM-1. This result is understandable in the framework of the microporous, sponge-like structure of PIM-1 providing a higher compressibility at a length scale defined by the lowest q value considered, than the denser Matrimid with no intrinsic microporosity.

Annealing of PIM-1 at elevated temperatures leads to a shift of the Boson Peak to higher frequencies compared to the unannealed case. Also, this result is related to shrinking of the microporous structure leading to a denser structure compared to the freshly prepared sample. This line of argument is supported by evidence from X-ray scattering observations.

In future work, further neutron scattering experiments will be carried out on other polymers with intrinsic microporosity to obtain a more complete picture.

\section{Acknowledgments:}

We thank the Maier-Leibnitz Zentrum (MLZ) at Garching for enabling the neutron scattering experiments. Dr. N. Konnertz and T. Rybak are acknowledged for their help in sample preparation. Dr W. Harrison is supported by EPSRC grant EP/K016946/1 "Graphene-based membranes". 


\section{References:}

1 P. M. Budd, B. S. Ghanem, S. Makhseed, N. B. McKeown, K. J. Msayib and C. E. Tattershall, Chem. Commun. (Cambridge, U. K.), 2004, 0, 230-231.

2 P. M. Budd, K. J. Msayib, C. E. Tattershall, B. S. Ghanem, K. J. Reynolds, N. B. McKeown and D. Fritsch, J. Membr. Sci., 2005, 251, 263-269.

3 K. Nagai, T. Masuda, T. Nakagawa, B. D. Freeman and I. Pinnau, Prog. Polym. Sci., 2001, 26, 721-798.

$4 \quad$ R. Swaidan, B. Ghanem and I. Pinnau, ACS Macro Lett., 2015, 4, 947-951.

$5 \quad$ Y. Yampolskii, Macromolecules, 2012, 45, 3298-3311.

$6 \quad$ P. M. Budd, N. B. McKeown, B. S. Ghanem, K. J. Msayib, D. Fritsch, L. Starannikova, N. Belov, O. Sanfirova, Y. Yampolskii and V. Shantarovich, J. Membr. Sci., 2008, 325, 851-860.

7 A. Bos, I. G. M. Pünt, M. Wessling and H. Strathmann, Sep. Purif. Technol., 1998, 14, 27-39.

8 J. W. Simmons and O. M. Ekiner, Polyimide and polyamide-imide gas separation membranes 1993, US5232472 A.

9 N. Konnertz, M. Böhning and A. Schönhals, Polymer, 2016, 90, 89-101.

10 H. Yin, P. Chapala, M. Bermeshev, A. Schönhals and M. Böhning, ACS Macro Lett., 2017, 6, 813-818.

11 C. H. Lau, P. T. Nguyen, M. R. Hill, A. W. Thornton, K. Konstas, C. M. Doherty, R. J. Mulder, L. Bourgeois, A. C. Y. Liu, D. J. Sprouster, J. P. Sullivan, T. J. Bastow, A. J. Hill, D. L. Gin and R. D. Noble, Angew. Chem., Int. Ed., 2014, 53, 5322-5326.

12 S. Harms, K. Rätzke, F. Faupel, N. Chaukura, P. M. Budd, W. Egger and L. Ravelli, J. Adhes., 2012, 88, 608-619.

13 W. F. Yong, K. H. A. Kwek, K.-S. Liao and T.-S. Chung, Polymer, 2015, 77, 377-386.

14 N. Konnertz, Y. Ding, W. J. Harrison, P. M. Budd, A. Schönhals and M. Böhning, J. Membr. Sci., 2017, 529, 274-285.

15 N. Konnertz, Y. Ding, W. J. Harrison, P. M. Budd, A. Schönhals and M. Böhning, ACS Macro Lett., 2016, 5, 528-532.

16 A. T. DiBenedetto and D. R. Paul, J. Polym. Sci., Part A: Gen. Pap., 1964, 2, $1001-$ 1015.

17 A. Y. Alentiev and Y. P. Yampolskii, J. Membr. Sci., 2002, 206, 291-306.

18 A. A. Gusev, S. Arizzi, U. W. Suter and D. J. Moll, J. Chem. Phys., 1993, 99, 22212227.

19 D. Hofmann, L. Fritz, J. Ulbrich, C. Schepers and M. Böhning, Macromol. Theory Simul., 2000, 9, 293-327.

20 R. Inoue, T. Kanaya, Y. Hu, T. Masuda, K. Nishida and O. Yamamuro, Polymer, 2014, 55, 182-186.

21 R. Inoue, T. Kanaya, T. Masuda, K. Nishida and O. Yamamuro, Macromolecules, 2012, 45, 6008-6014.

22 M. Böhning, N. Hao and A. Schönhals, J. Polym. Sci., Part B: Polym. Phys., 2013, 51, 1593-1597.

23 M. Heuchel, D. Fritsch, P. M. Budd, N. B. McKeown and D. Hofmann, J. Membr. Sci., 2008, 318, 84-99.

24 O. Hölck, M. Böhning, M. Heuchel, M. R. Siegert and D. Hofmann, J. Membr. Sci., 2013, 428, 523-532.

25 N. B. McKeown and P. M. Budd, Macromolecules, 2010, 43, 5163-5176.

26 T. Emmler, K. Heinrich, D. Fritsch, P. M. Budd, N. Chaukura, D. Ehlers, K. Rätzke and F. Faupel, Macromolecules, 2010, 43, 6075-6084. 
27 J. Rouquerol, D. Avnir, C. W. Fairbridge, D. H. Everett, J. M. Haynes, N. Pernicone, J. D. F. Ramsay, K. S. W. Sing and K. K. Unger, Pure Appl. Chem., 1994, 66, 17391758.

28 R. Zallen, The physics of amorphous solids, Wiley, New York, 1983.

29 V. K. Malinovsky, V. N. Novikov and A. P. Sokolov, J. Non-Cryst. Solids, 1987, 90, 485-488.

30 U. Buchenau, Y. M. Galperin, V. L. Gurevich, D. A. Parshin, M. A. Ramos and H. R. Schober, Phys. Rev. B: Condens Matter Mater. Phys., 1992, 46, 2798-2808.

31 B. B. Laird and H. R. Schober, Phys. Rev. Lett., 1991, 66, 636-639.

32 S. N. Taraskin, Y. L. Loh, G. Natarajan and S. R. Elliott, Phys. Rev. Lett., 2001, 86, $1255-1258$.

33 W. Schirmacher, G. Diezemann and C. Ganter, Phys. Rev. Lett., 1998, 81, 136-139.

34 R. Milkus and A. Zaccone, Phys. Rev. B, 2016, 93, 094204.

35 A. Schönhals, R. Zorn and B. Frick, Polymer, 2016, 105, 393-406.

36 R. Zorn, M. Mayorova, D. Richter, A. Schönhals, L. Hartmann, F. Kremer and B. Frick, AIP Conf. Proc., 2008, 982, 79-84.

37 T. Asthalter, M. Bauer, U. van Bürck, I. Sergueev, H. Franz and A. I. Chumakov, Eur. Phys. J. E: Soft Matter Biol. Phys., 2003, 12, S9-12.

A. Schönhals, H. Goering, C. Schick, B. Frick and R. Zorn, Colloid Polym. Sci., 2004, 282, 882-891.

39 R. Zorn, Phys. Rev. B: Condens. Matter Mater. Phys., 2010, 81, 054208.

40 P. Li, T. S. Chung and D. R. Paul, J. Membr. Sci., 2013, 432, 50-57.

41 J. Ahn, W. J. Chung, I. Pinnau, J. S. Song, N. Y. Du, G. P. Robertson and M. D. Guiver, J. Membr. Sci., 2010, 346, 280-287.

42 Heinz Maier-Leibnitz Zentrum. (2015). TOFTOF: Cold neutron time-of-flight spectrometer. Journal of large-scale research facilities, 1, A15. http://dx.doi.org/10.17815/jlsrf-1-40

$43 \mathrm{http}: / / \mathrm{www} . i 11 . e u /$ instruments-support/computing-forscience/cs-software/allsoftware/tofhr/inx/.

44 J. Filik, A. W. Ashton, P. C. Y. Chang, P. A. Chater, S. J. Day, M. Drakopoulos, M. W. Gerring, M. L. Hart, O. V. Magdysyuk, S. Michalik, A. Smith, C. C. Tang, N. J. Terrill, M. T. Wharmby and H. Wilhelm, J. Appl. Cryst., 2017, 50, 959-966.

45 B. R. Pauw, A. J. Smith, T. Snow, N. J. Terrill and A. F. Thünemann, J. Appl. Cryst., submitted, arXiv:1706.06769.

46 A. G. McDermott, G. S. Larsen, P.M. Budd, C. M. Colina and J. Runt, Macromolecules, 2011, 44, 14-16.

47 A. G. McDermott, P.M. Budd, N. B. McKeown, C. M. Colina and J. Runt, J. Mater. Chem. A., 2014, 2, 11742-11752.

48 R.-J. Roe, Methods of X-Ray and Neutron Scattering in Polymer Science; Oxford University Press: New York, 2000.

49 M. Bée, Quasielastic neutron scattering. Principles and applications in solid state chemistry, biology and materials science, Adam Hilger, 1988.

50 S. W. Lovesey, Theory of Neutron Scattering from Condensed Matter, Oxford University, New York, 1987, vol. 1, p. 121.

R. Zorn, L. Hartmann, B. Frick, D. Richter and F. Kremer, J. Non-Cryst. Solids, 2002, 307, 547-554.

52 B. Frick, U. Buchenau and D. Richter, Colloid and Polymer Sci., 1995, 273, 413-420

53 I. Rintaro and T. Kanaya, Heterogeneous Dynamics of Polymer Thin Films as Studied by Neutron Scattering. In Glass Transition, Dynamics and Heterogeneity of Polymer 
Thin Films, 1st ed.; T. Kanaya, Ed.; Advances in Polymer Science, Vol. 252; Springer: Berlin, 2013; pp 107-140.

54 T. Kanaya, I. Tsukushi, K. Kaji, B. J. Gabrys, S. Bennigton and H. Furuya, Phys. Rev. $B, 2001,64,144202$.

55 W. Schirmacher, B. Schmid, C. Tomaras, G. Viliani, G. Baldi, G. Ruocco and T. Scopigno, Phys. Status Solidi C, 2008, 5, 862-866.

56 M. T. Cicerone and C. L. Soles, Biophys. J., 2004, 86, 3836-3845.

57 C. Krause, R. Zorn, F. Emmerling, J. Falkenhagen, B. Frick, P. Huber and A. Schönhals, Phys. Chem. Chem. Phys., 2014, 16, 7324-7333.

$58 \quad$ W. Götze and L. Sörgren, Rep. Prog. Phys. 1992, 50, 241-376.

59 P. M. Budd, E. S. Elabas, B. S. Ghanem, S. Makhseed, N. B. McKeown, K. J . Msayib, C. E. Tattershall and D. Wang, Adv. Mater. 2004. 16, 456-459.

60 A.C. Comer, D.S. Kalika, B.W. Rowe, B.D. Freeman, D.R. Paul, Polymer 2009, 50, 891897.

61 A. I. Chumakov, G. Monaco, A. Monaco, W. A. Crichton, A. Bosak, R. Rüffer, A. Meyer, F. Kargl, L. Comez, D. Fioretto, H. Giefers, S. Roitsch, G. Wortmann, M. H. Manghnani, A. Hushur, Q. Williams, J. Balogh, K. Parlinski, P. Jochym, and P. Pickarz, Phys. Rev. Lett., 2011, 106, 225501

62 V. K. Malinovsky and A. P. Sokolov, Solid State Commun., 1986, 57, 757-761.

63 V. K. Malinovsky, V. N. Novikov and A. P. Sokolov, Chem. Phys. Lett., 1988, 143, 111-114.

64 L. Hong, V. N. Novikov and A. P. Sokolov, Phys. Rev. E, 2011, 83, 061508.

65 F. Scarponi, L. Comez, D. Fioretto and L. Palmieri, Phys. Rev. B 2004, 70, 054203 